\section{Effects of Early Rituximab Retreatment in Rheumatoid Arthritis Patients with an Inadequate Response After the First Cycle: Retrospective Arthritis Cohort Study}

\section{To the Editor:}

The therapeutic options for rheumatoid arthritis (RA) have been significantly improved by the introduction of novel biological agents ${ }^{1}$. In this context the chimeric anti-CD20 monoclonal antibody rituximab (RTX) was approved for treatment of active and resistant RA in Germany in 2006. RTX was shown to represent a safe and effective treatment option over several courses leading to selective and transient depletion of the CD20+ B cell population ${ }^{2-4}$. However, to date the optimal timepoint for retreatment has not been defined exactly, and longterm treatment strategies using RTX have not been established.

Our observational cohort study was performed to compare different treatment strategies using RTX and especially the effects of early retreatment within 4 to 6 months after first drug administration with a retreatment interval of more than 6 months in patients with RA (Figure 1). Baseline characteristics of the 39 RA patients are shown in Table 1. The respective comorbidities included overlapping chronic rheumatic diseases in 8 cases (systemic lupus erythematosus, cutaneous lupus erythematosus, scleroderma, ankylosing spondylitis, and 4 patients with secondary Sjögren's syndrome). Patient histories revealed 2 cases of previous mycobacterial infections, a case of chronic hepatitis $\mathrm{C}$ infection, and 3 cases of malignant diseases.

For the first course of RTX, 39 patients received 2 doses of $1000 \mathrm{mg}$ RTX (in one patient $2 \times 500 \mathrm{mg}$ RTX). For subsequent treatment courses, 27 patients received a second, 14 patients a third, 4 patients a fourth, and one patient a fifth RTX administration with the recommended premedication.

Four months after the first course of RTX, patients were classified according to their short-term behavior as classical responders [reduction of Disease Activity Score 28-joint count (DAS28) by $>1.2$ and retherapy after 6 months], as nonresponders (no relevant reduction of DAS28 and retherapy after 6 months), and as patients requiring short-term retherapy (rether- apy between 4 and 6 months after first RTX course). The reason for early retherapy was primary nonresponse to RTX in 3 cases and early relapses after significant response in the remaining 3 cases (Figure 1).

Subsequently, followup data of the same patients were evaluated for their DAS28 response and reduction of C-reactive protein (CRP) and erythrocyte sedimentation rate (ESR) 4 months after the second RTX course.

The results were as follows. Longterm efficacy was evaluated 4 months after baseline of the second RTX cycle. In the group of classical responders $(n=19)$, after the first RTX course, a significant reduction was also evident in the DAS28, from $5.82 \pm 0.98$ to $3.8 \pm 1.43$ ( $\mathrm{p}<0.0002$, Mann-Whitney test), in the longterm evaluation. Further, a significant reduction of ESR (from $33.8 \pm 16.9 \mathrm{~mm} / \mathrm{h}$ to $17.0 \pm 10.9 \mathrm{~mm} / \mathrm{h} ; \mathrm{p}<0.0096$ ) and a numerical reduction of CRP (from $5.3 \pm 7.9 \mathrm{mg} / \mathrm{dl}$ to $1.2 \pm 1.7 \mathrm{mg} / \mathrm{dl}$; p $<0.0671$ ) were observed (Figure 2).

In the group of patients defined as nonresponders $(n=14)$ after the first RTX course, a significant reduction of DAS28 from $5.1 \pm 1.2$ to $3.4 \pm 1.45$ ( $p<0.0109$ ) was also observed 4 months after the second cycle of RTX. However, the reduction of the CRP and ESR values was not significant compared to baseline levels (Figure 3).

In the group of patients with early RTX retreatment between months 4 and $6(\mathrm{n}=6)$ after the first cycle, there was a significant reduction of DAS28, from $6.5 \pm 1.0$ to $2.77 \pm 0.95(\mathrm{p}<0.0043)$, as well as a significant reduction of CRP values $(7.45 \pm 5.65 \mathrm{mg} / \mathrm{dl}$ to $1.62 \pm 2.47 \mathrm{mg} / \mathrm{dl} ; \mathrm{p}<$ 0.0317 ) and ESR (from $51.2 \pm 26.9 \mathrm{~mm} / \mathrm{h}$ to $4.0 \pm 3.26 \mathrm{~mm} / \mathrm{h} ; \mathrm{p}<0.0095$; Figure 4).

In summary, our data show retreatment of classical RTX responders after 6 months was associated with a good longterm result in DAS28 improvement as well as CRP and ESR reduction. In patients characterized as primary nonresponders, retreatment after 6 months showed limited efficacy, with relevant reduction of DAS28 but not in CRP and ESR levels.

Notably, our preliminary results also indicate that a favorable effect can be achieved by early retreatment of nonresponders to a first RTX cycle. Due to the limited number of patients in our study, further investigations in larger cohorts are required to substantiate this observation.

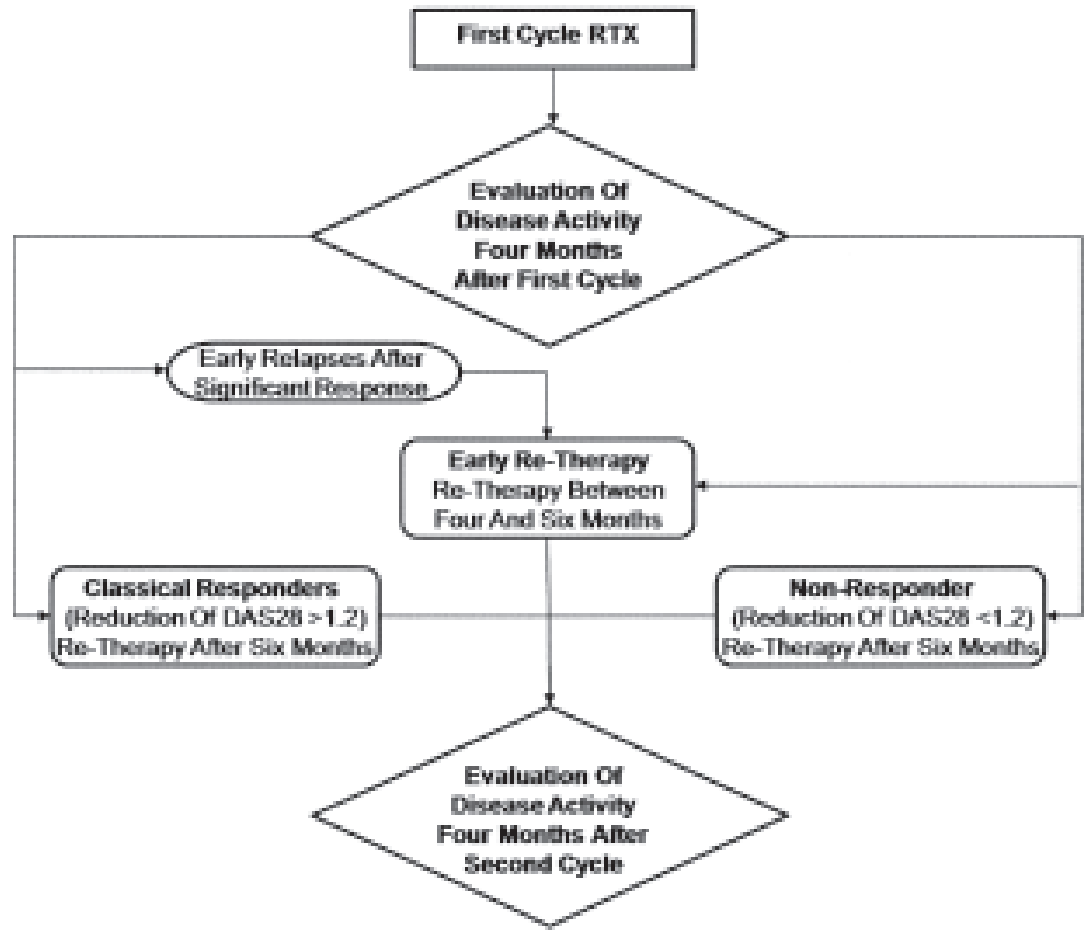

Figure 1. Progress of patients through treatment. 
Table 1. Baseline demographic characteristics of the observational cohort. Data are mean \pm SD or number (\%).

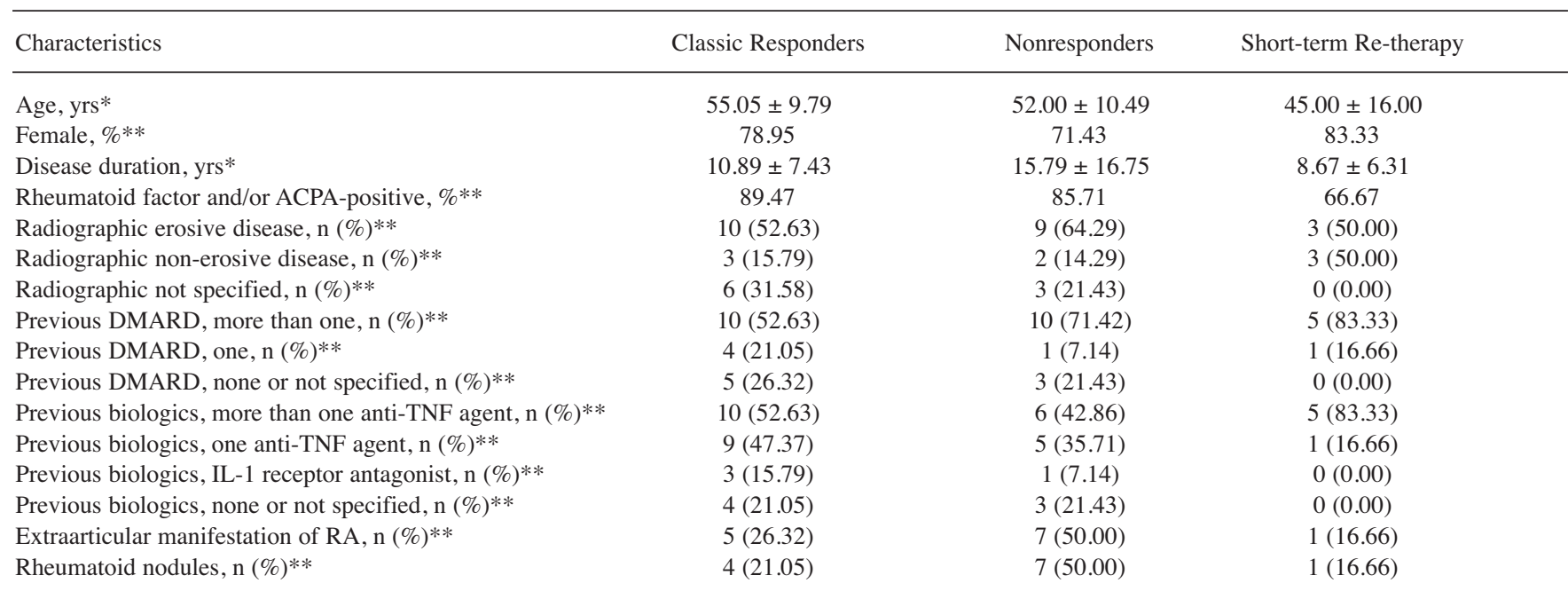

* No significant differences between groups (Mann-Whitney U test); ** No significant differences between groups (chi-square test). ACPA: anti-citrullinated protein/peptide antibodies; DMARD: disease-modifying antirheumatic drug; IL: interleukin; TNF: tumor necrosis factor.

DAS28

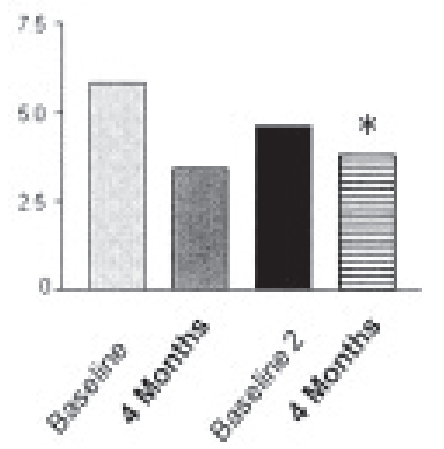

CRP mg/dl

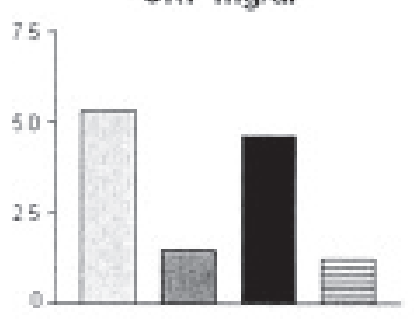

$(n=19)$

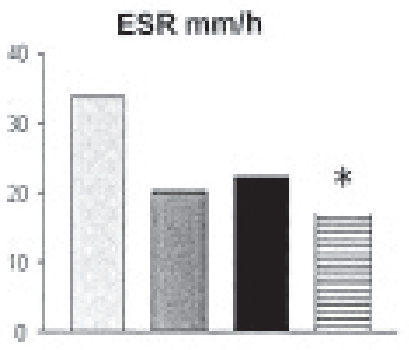

Figure 2. Clinical characteristics of classical responders to treatment: reduction of DAS28 by $>1.2$ and retherapy after 6 months. *Significant reduction.

DAS28

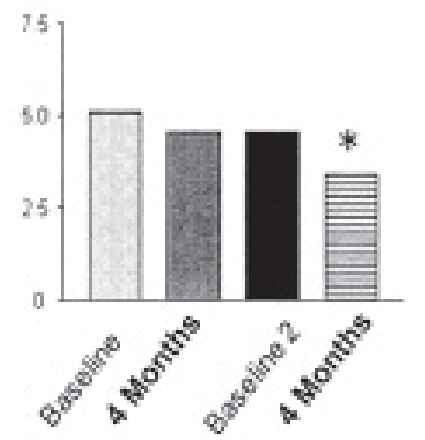

CRP mg/dl

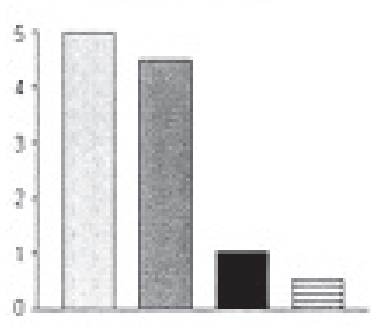

$(n=14)$

Figure 3. Clinical characteristics of nonresponders: no relevant reduction of DAS28 and retherapy after 6 months. *Significant reduction. 
DAS28

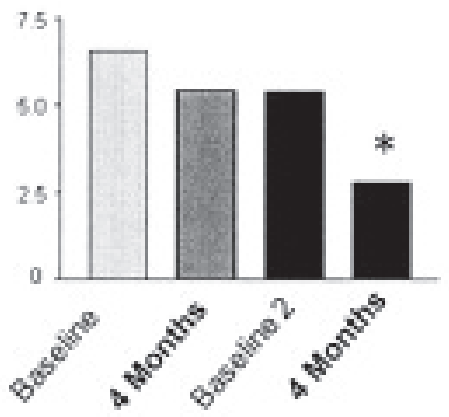

CRP mg/dl

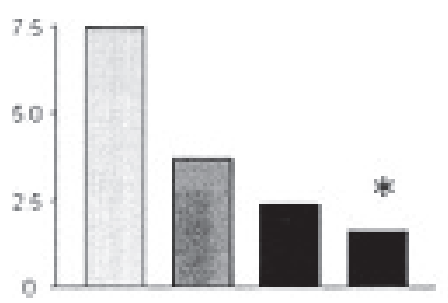

$(n=6)$
ESR $\mathrm{mm} / \mathrm{h}$

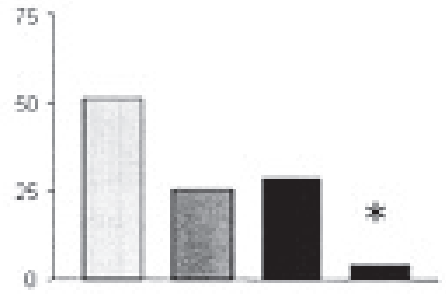

Figure 4. Clinical characteristics after short-term retherapy (between 4 and 6 months after first RTX course). *Significant reduction.

HANS BASTIAN, Department of Rheumatology and Clinical Immunology, Charité - Universitätsmedizin Berlin, Campus Mitte; SILKE ZINKE, MD, Rheumatological Office, Berlin; KARL EGERER, $\mathrm{PhD}$, Department of Rheumatology and Clinical Immunology, Charité - Universitätsmedizin Berlin, Campus Mitte; STEFFEN BREUER; FRANK SAFARI, Roche Pharma AG, Grenzach-Wyhlen; GERD-RÜDIGER BURMESTER, MD; EUGEN FEIST, MD, Department of Rheumatology and Clinical Immunology, Charité -

Universitätsmedizin Berlin, Campus Mitte, Berlin, Germany. Address correspondence to H. Bastian, Department of Rheumatology and Clinical Immunology, Charité - Universitätsmedizin Berlin, Campus Mitte, Charitéplatz 1, 10117 Berlin, Germany. E-mail: hans.bastian@charite.de Supported by Roche Germany. Dr. Feist and Dr. Burmester were consultants and speakers for and received research support from Roche Pharma AG.

\section{REFERENCES}

1. Alamanos Y, Drosos AA. Epidemiology of adult rheumatoid arthritis. Autoimmun Rev 2005;4:130-6.
2. Edwards JC, Cambridge G. Sustained improvement in rheumatoid arthritis following a protocol designed to deplete B lymphocytes. Rheumatology 2001;40:205-11.

3. Cohen SB, Emery P, Greenwald MW, Dougados M, Furie RA, Genovese MC, et al. Rituximab for rheumatoid arthritis refractory to anti-tumor necrosis factor therapy: Results of a multicenter, randomized, double-blind, placebo-controlled, phase III trial evaluating primary efficacy and safety at twenty-four weeks. Arthritis Rheum 2006;54:2793-806.

4. Emery P, Fleischmann R, Filipowicz-Sosnowska A, Schechtman L, Szczepanski L, Kavanaugh A, et al. The efficacy and safety of rituximab in patients with active rheumatoid arthritis despite methotrexate treatment: results of a phase IIB randomized, double-blind, placebo-controlled, dose-ranging trial. Arthritis Rheum 2006;54:1390-400.

J Rheumatol 2010;37:5; doi:10.3899/jrheum.091127 